\title{
Delaying progression to type 2 diabetes among high-risk Spanish individuals is feasible in real-life primary healthcare settings using intensive lifestyle intervention
}

\author{
B. Costa • F. Barrio • J.-J. Cabré • J.-L. Piñol • X. Cos • \\ C. Solé • B. Bolíbar • J. Basora • C. Castell • \\ O. Solà-Morales • J. Salas-Salvadó • J. Lindström • \\ J. Tuomilehto • The DE-PLAN-CAT Research Group
}

Received: 30 July 2011 / Accepted: 20 January 2012 /Published online: 10 February 2012

(C) Springer-Verlag 2012

\begin{abstract}
Aims/hypothesis To assess the feasibility and effectiveness of an active real-life primary care lifestyle intervention in preventing type 2 diabetes within a high-risk Mediterranean population.

Methods A prospective cohort study was performed in the setting of Spanish primary care. White-European individuals without diabetes aged $45-75$ years $(n=2,054)$ were screened using the Finnish Diabetes Risk Score (FINDRISC) and a subsequent $2 \mathrm{~h}$ OGTT. Where feasible, high-risk individuals who were identified were allocated sequentially to standard care, a group-based or an individual
\end{abstract}

A complete list of members of the Coordinating Committee is given in the Appendix.

B. Costa $\cdot$ F. Barrio $\cdot$ J.-J. Cabré $\cdot$ J.-L. Piñol $\cdot$ X. Cos $\cdot$ C. Solé $\cdot$

B. Bolíbar $\cdot$ J. Basora

Jordi Gol Primary Care Research Institute (Diabetes and

Metabolism), Catalan Health Institute,

Reus, Tarragona-Barcelona, Spain

B. Costa $(\bowtie)$

Centre Diabetis Salou,

Via Roma 32,

43840 Salou, Tarragona, Spain

e-mail: costaber@gmail.com

C. Castell

Department of Health, Generalitat de Catalunya,

Barcelona, Spain

O. Solà-Morales

Catalan Agency for Health Information, Generalitat de Catalunya,

Barcelona, Spain

J. Salas-Salvadó

Nutrition Unit,

Institute of Health Research Pere Virgili (CIBERobn),

Reus, Spain level intervention (intensive reinforced DE-PLAN [Diabetes in Europe-Prevention using Lifestyle, Physical Activity and Nutritional] intervention). The primary outcome was the development of diabetes according to WHO criteria. Analyses after 4-year follow-up were performed based on the intention-to-treat principle with comparison of standard care and the combined intervention groups.

Results The standard care $(n=219)$ and intensive intervention $(n=333)$ groups were comparable in age $(62.0 / 62.2$ years $)$, sex (64.4/68.2\% women), BMI $\left(31.3 / 31.2 \mathrm{~kg} / \mathrm{m}^{2}\right)$, FINDRISC score (16.2/15.8 points), fasting $(5.3 / 5.2 \mathrm{mmol} / \mathrm{l}), 2 \mathrm{~h}$ plasma glucose $(7.1 / 6.9 \mathrm{mmol} / \mathrm{l})$ and self-reported interest to make

J. Lindström

Diabetes Prevention Unit,

National Institute for Health and Welfare,

Helsinki, Finland

J. Tuomilehto

Department of Public Health, University of Helsinki,

Helsinki, Finland

J. Tuomilehto

South Ostrobothnia Central Hospital,

Seinajoki, Finland

J. Tuomilehto

Department of Clinical and Preventive Medicine,

Danube-University Krems,

Krems, Austria 
lifestyle changes at baseline. Diabetes was diagnosed in 124 individuals: $63(28.8 \%)$ in the standard care group and 61 $(18.3 \%)$ in the intensive intervention group. During a 4.2-year median follow-up, the incidences of diabetes were 7.2 and 4.6 cases per 100 person-years, respectively $(36.5 \%$ relative risk reduction, $p<0.005$ ). The number of participants needed to be treated by intensive intervention for 4 years to reduce one case of diabetes was 9.5 .

Conclusions/interpretation Intensive lifestyle intervention is feasible in a primary care setting and substantially reduces diabetes incidence among high-risk individuals.

Clinical trial registration: ClinicalTrial.gov NCT01519505 Funding: Commission of the European Communities, Institute of Health Carlos III, Spanish Ministry of Health and Department of Health, Generalitat de Catalunya.

Keywords Diabetes prevention · FINDRISC · Incidence . Lifestyle intervention · Primary care

$\begin{array}{ll}\text { Abbreviations } \\ \text { FINDRISC } & \text { Finnish Diabetes Risk Score } \\ \text { FPG } & \text { Fasting plasma glucose } \\ \text { IFCC } & \text { International Federation of Clinical Chemistry } \\ \text { IFG } & \text { Impaired fasting glucose } \\ \text { IGT } & \text { Impaired glucose tolerance } \\ \text { NGSP } & \text { National Glycohemoglobin Standardization } \\ & \text { Program } \\ \text { 2hPG } & 2 \text { h Plasma glucose } \\ \text { RRR } & \text { Relative risk reduction }\end{array}$

\section{Introduction}

Type 2 diabetes is an important preventable disease and a growing public health problem. Based on clinical trials, it is known that type 2 diabetes development can be prevented or delayed by intensive lifestyle intervention among high-risk people. However, developing a diabetes prevention strategy that applies to the general population is challenging and requires political commitment [1-3].

When planning national diabetes prevention measures, people at high risk of developing type 2 diabetes should be targeted with lifestyle modification interventions through a stepwise high-risk approach tailored to the specific local situation [2]. A key issue is translation of diabetes prevention research into practice. In fact, community-based evaluations in a range of countries are essential to learn about the viability and effectiveness of programmes designed and implemented locally [4].

The present study aimed to assess the feasibility, and subsequently the effectiveness, of an active real-life primary care strategy to prevent type 2 diabetes in high-risk Spanish individuals using existing public healthcare resources.

\section{Methods}

The methodology from a public health programme, DEPLAN (Diabetes in Europe-Prevention using Lifestyle, Physical Activity and Nutritional intervention), developed in Catalonia (Spain) was used for study purposes [5]. A multidisciplinary Steering Committee was established with representatives from each primary care centre to implement a single common protocol as well as a curriculum for the training of prevention managers (general practitioners and nurses). These participating centres were selected in a stratified manner and covered all primary care services for 315,703 inhabitants ( $4.5 \%$ of the population in Catalonia). All participating professionals were certified after several training meetings. The supplementary resources used were recorded and partially funded. The research ethics committee board at the Jordi Gol Research Institute (Barcelona, Spain) approved the protocol and all participants gave written informed consent.

White-European individuals without diabetes aged 4575 years were evaluated by general practitioners in 18 primary healthcare centres. The participants were consecutively recruited from a random list from the computerised public healthcare system to obtain a representative sample of the population assigned to each centre. All individuals with severe psychiatric disease, chronic kidney and liver disease or blood disorders were excluded.

The first screening used the Spanish version of the Finnish Diabetes Risk Score (FINDRISC), a well-validated eight-item European questionnaire related to diabetes risk factors (the most recent version used ranged from 0 to 26 points) characterising individuals according to their future risk of type 2 diabetes as follows: $(<7$ points [low], $7-11$ [slightly elevated], 12-14 [moderate], 15-20 [high], $>20$ [very high]) [3, 6] (www.diabetes.fi). The questionnaire collected information about age, sex, weight and height (BMI), waist circumference, use of concomitant blood pressure medication, history of high blood glucose disorders, physical activity, family history of diabetes and daily consumption of vegetables, fruits or berries. Both questionnaire and European guidelines recommend that people with scores $>14$ should have blood tests for detecting diabetes [3-6]. Body weight and height were measured in light clothing, without shoes. Waist circumference was measured midway between the lowest rib and the iliac crest. Anthropometric variables were determined by trained nurses.

The second screening was carried out using a $2 \mathrm{~h} 75 \mathrm{~g}$ OGTT according to WHO standards, with measurements of fasting and $2 \mathrm{~h}$ postload plasma glucose. All participants were asked to have a screening OGTT as part of the protocol. Participants with FINDRISC scores $\leq 14$ were offered an OGTT on a voluntary basis. A second OGTT to confirm a diagnosis of diabetes based on the first OGTT was 
recommended in the study protocol. These measurements were repeated at the yearly follow-up visits to determine type 2 diabetes incidence. The lipid profile and $\mathrm{HbA}_{1 \mathrm{c}}$ determinations were performed simultaneously. Three main diagnostic categories (normal, prediabetes and diabetes) were defined based on $2 \mathrm{~h}$ postload glucose $(<7.8 ; 7.8-$ $11.0 \mathrm{mmol} / \mathrm{l}$ and/or fasting plasma glucose [6.1-6.9 mmol/1]; and $>11.1 \mathrm{mmol} / \mathrm{l}$, respectively) [7]. The plasma glucose and lipid profile determinations were carried out using a uniform glucose oxidase-peroxidase and a cholesterol oxidase-phenol aminophenazone (CHOD-PAP) method, respectively. The $\mathrm{HbA}_{1 \mathrm{c}}$ assay was a standardised HPLC assay aligned to the Diabetes Control and Complications Trial in all laboratories [8]. The intra- and interassay coefficients of variation for all assays ranged from $2 \%$ to $3 \%$.

People were eligible for the lifestyle intervention only if they had had an OGTT, did not have diabetes and had either or both of a FINDRISC score $>14$ or prediabetes defined using WHO criteria for fasting or $2 \mathrm{~h}$ glucose. Prior to any intervention, the participants completed a 46-item basic questionnaire provided by the European DE-PLAN/IMAGE project adapted to the Spanish language and habits $[4,5]$. This survey was focused on dietary and physical exercise behaviour to detect participants' baseline status and possible future changes. In view of the present evaluation, particular attention was paid to eight specific items aimed at assessing the individual self-reported interest in introducing lifestyle changes.

The protocol consisted of two interventions (standard care or intensive) and two steps (start and reinforcement). In turn, intensive intervention was delivered individually or in groups. The following targets for lifestyle intervention were suggested: no more than $30 \%$ of daily energy from fat, no more than $10 \%$ of energy from saturated fat, at least $3.6 \mathrm{~g} / 1,000 \mathrm{~kJ}(15 \mathrm{~g} / 1,000 \mathrm{kcal})$ of fibre, at least $30 \mathrm{~min} /$ day of moderate physical activity and at least an arbitrary but realistic 3\% weight reduction. The participating centres were asked to assign individuals who agreed to take part in the programme consecutively to the standard care intervention, the individual intensive lifestyle intervention and the group intensive intervention if it was feasible in their routine clinical practice. Before measurements were taken, people allocated to the intensive intervention were given the choice of group or individual sessions.

Participants in the standard care intervention each received general information on diet, cardiovascular health and the risk of type 2 diabetes, without an individualised programme. The process was reinforced by taking advantage of subsequent visits to the centre, as in standard healthcare practice.

The intensive group intervention consisted of a $6 \mathrm{~h}$ educational programme scheduled in two to four sessions with five to 15 participants, who also received specific training materials. The methods were adapted to the experience, needs and skills available (empowerment) based on motivation, peer support and positive feedback. The cornerstones of the contents were: (1) what type 2 diabetes is and what it means to be at risk; (2) the Mediterranean diet and nutritional advice based on the Prevención con Dieta Mediterránea-Mediterranean Diet Adherence Screener (PREDIMED MEDAS) questionnaire as a tool to increase adherence to the Mediterranean diet during the follow-up [9]; (3) physical activity and its beneficial health effects; and (4) tobacco advice (in the centres with smokers in the intervention group). As for the individual intensive intervention group, the methods used were similar but individually delivered. To maintain motivation for preventive lifestyle changes, regular contact by phone or text message was programmed at least once every 6-8 weeks. Process-based evaluation of the individual risk and response was provided to encourage the lifestyle modification.

An effort was made to ascertain the type 2 diabetes status in people who discontinued the programme prematurely as well. These individuals were independently identified at the end of the follow-up by their primary care teams or the group responsible for data treatment; an additional blood test for each of these individuals was sought when required. Finally, diabetes diagnoses of all individuals who discontinued the protocol were ascertained and included in the intention-to-treat analysis.

Statistical analyses were conducted using SPSS version 15.0 for Windows (SPSS Inc., Chicago, IL, USA). The sample size was calculated based on available data on diabetes incidence in the high-risk Catalan population [10]. Consequently, it was assumed that the mean annual incidence in the standard care intervention group would be $7.5 \%$ and the mean expected incidence in the group of maximum impact (intensive intervention group) would be $3.25 \%$ ( $50 \%$ reduction in the yearly rate). Basic hypotheses for calculations were: $35 \%$ positive high-risk screenees by the FINDRISC, $10 \%$ negative screenees with prediabetes diagnostic criteria at OGTT, $20 \%$ of individuals having undiagnosed diabetes and a theoretical distribution between participants in the standard care, individualised intensive and group intensive interventions close to 1:1:1. Allowing for a discontinuation rate of $30 \%$, it was assumed that no less than 1,650 people should be included in the screening phase to include at least 550 participants in the lifestyle intervention (type $1 /$ type 2 error $5 \% / 20 \%$ ).

Multiple comparisons of significant differences among groups were carried out by one-way ANOVA and/or by Student's $t$ test. The level of statistical significance was set as $p<0.05$ for all analyses. Person-years were the sum of time under follow-up for all participants before diabetes diagnosis or end of follow-up if diabetes did not develop during the study period. We estimated the cumulative 
incidence and the probability of remaining diabetes-free in each intervention group using Kaplan-Meier survival curves. Participants who discontinued the study protocol were considered to be at risk for diabetes until their last visit, at which point data were censored. The difference between the curves was evaluated with the two-sided logrank test. The Cox proportional hazards model was used to estimate the HR for development of diabetes. The analyses were adjusted for intervention group, sex, age and baseline blood test results: $2 \mathrm{hPG}$ or the corresponding prediabetes interval (IGT 7.8-11.0 mmol/1), FPG or the corresponding prediabetes interval (IFG 6.1-7.0 mmol/l), $\mathrm{HbA}_{1 \mathrm{c}}$ and lipid profile values. First, the biochemical measurements were included in the model and then omitted, keeping the FINDRISC items separate. The 95\% CI were also estimated. All comparisons were based on the intention-to-treat principle.

\section{Results}

In all, 2,054 individuals were screened by the FINDRISC (81\% response) and $1,192(58 \%)$ received an OGTT. Of these, $624(52.3 \%)$ were classified as being at high type 2 diabetes risk by the FINDRISC $(n=347)$, the OGTT $(n=106)$ or both tests $(n=171)$, and were offered the lifestyle intervention (Fig. 1). Finally, 552 (88.3\%) agreed to participate in the intervention programme. In two of the 18 sites, the intensive intervention was not available and 51 individuals (9.3\%) were allocated directly to the standard care group. In the remaining 16 centres, the consecutive assignment procedure was feasible $(n=501$ individuals, $90.7 \%)$ and 168 participants $(33.6 \%)$ were assigned to the standard care group, $171(34.1 \%)$ to the individual intensive intervention and $162(32.3 \%)$ to the group intensive intervention. After allowing personal choice between intensive approaches, 79 participants initially allocated to individual intensive intervention chose to swap to the group approach (46.2\%) and 11 participants initially allocated to group intervention chose to swap to the individual intensive approach (6.8\%). In short, a total of 219 (39.7\%) were assigned to standard care intervention and $333(60.3 \%)$ to intensive lifestyle intervention (Fig. 1). No statistical differences in the screening variables were found between individuals who accepted $(n=552)$ or refused $(n=73)$ the lifestyle intervention programme. The first individual was allocated in March 2006 and the last in December 2006; the study ended in December 2010. The median follow-up was 4.2 years (mean 3.8 years).

A total of $66.7 \%$ of participants were female, mean age was 62.1 years and mean BMI was $31.2 \mathrm{~kg} / \mathrm{m}^{2}$. The risk of diabetes assessed by the first screening test (FINDRISC score) was higher in women $(16.2 / 15.8$ points, $p<0.001)$. In contrast, the risk of diabetes found by the second screening test (OGTT) was higher in men both fasting $(5.4 / 5.1 \mathrm{mmol} / 1, p<0.001)$ and $2 \mathrm{~h}$ postload plasma glucose $(7.4 / 6.8, p<0.001)$. Despite these differences, the standard care and the intensive intervention groups were statistically comparable regarding all baseline findings, which reflected similar risk factor patterns (Table 1). In addition, no statistically significant differences between groups were found in self-reported interest to make lifestyle changes ( $p$ values ranging from 0.59 to 0.98 ).

During the study, 228 individuals (41.3\%) discontinued the study protocol: $102(46.6 \%)$ in the standard care intervention group and $126(37.8 \%)$ in the intensive group. Of these, 56 could not be contacted, 24 discontinued due to severe illness, three died and 145 withdrew for personal reasons (most alluding to problems in their work schedule or from rejection of the blood test). Mean annual discontinuation rate was slightly higher in the standard care group (12.6\% vs 9.7\%, $p=0.04)$. All participants discontinuing the study could be traced by direct personal contact or searching into their computerised medical records; we included 19 (8.4\%) diabetes-related diagnoses (11 individuals allocated into the standard care group and eight into the intensive group) in the intention-to-treat analysis. Of these, nine individuals were receiving specific diabetes treatment, four had been diagnosed at least by two blood tests and six had agreed to a blood retest according to the protocol.

Diabetes was diagnosed in a total of 124 participants: 63 $(28.8 \%)$ in the standard care group and 61 (18.3\%) in the intensive group. The absolute incidences of diabetes were 7.2 and 4.6 cases per 100 person-years, respectively $(p<0.005$, logrank test). Consequently, during the Catalan DE-PLAN programme, the risk of diabetes was reduced by $36.5 \%$ in the intensive intervention group compared with the standard care group. Similarly, the incidence of diabetes in participants with prediabetes (IFG and/or IGT) was 13.4 cases per 100 person-years in the standard care group and 8.9 cases per 100 person-years in the intensive group (33.6\% RRR, $p=0.01)$. Table 2 shows a similar baseline risk pattern between participants who progressed to diabetes or did not in the standard care and the intensive intervention study groups. Overall, 13 individuals were diagnosed as having diabetes by medical records and 111 participants on the basis of an OGTT, of whom 44 and 67 were subsequently confirmed by fasting and $2 \mathrm{~h}$ postload glucose values, respectively. The incidence of diabetes in the subgroup of participants directly assigned to standard care intervention was 8.2 cases per 100 person-years.

Diabetes incidence increased over time, but not proportionally. The cumulative incidence of diabetes was lower in the intensive group than in the standard care group with statistically significant differences from the third year. After 4 years, the cumulative incidences were $18.3 \%$ (95\% CI $14.3,22.9)$ and $28.8 \%(95 \%$ CI $22.9,35.3)$, respectively 


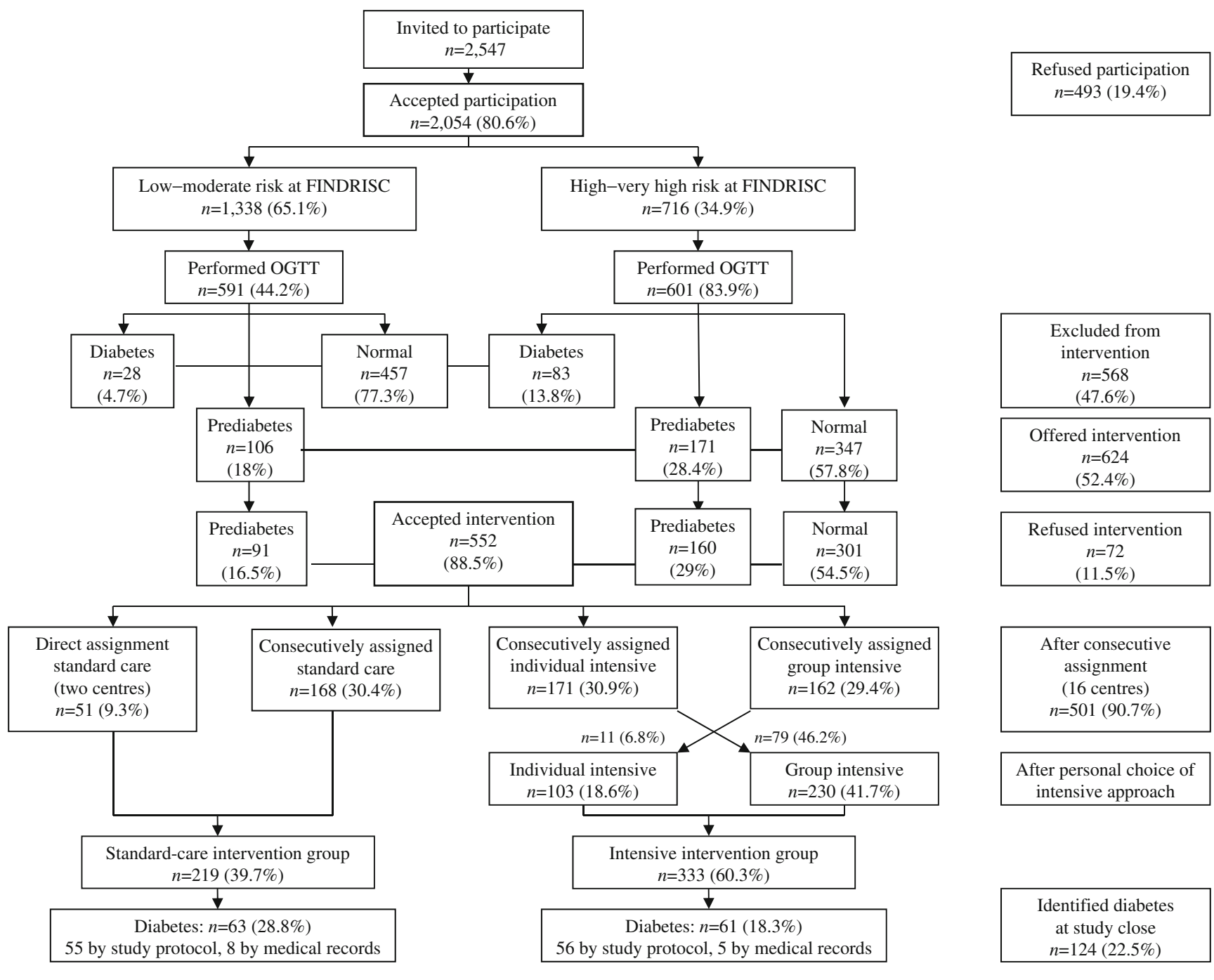

Fig. 1 Flow diagram describing the DE-PLAN-CAT study design, number of participants and the main outcomes by lifestyle intervention groups

(36.5\% RRR). The corresponding 4-year HR was 0.64 (95\% CI $0.47,0.87 ; p=0.004)$. The cumulative probability of remaining diabetes-free after 4 years was $81.9 \%$ (95\% CI $76,87.8)$ in the intensive group and $72.5 \%$ (95\% CI 68.4 , 76.6) in the standard care group (Fig. 2). The cumulative 4year incidence of diabetes in men was $21.7 \%$ in the intensive group $(95 \%$ CI $14.3,30.8)$ and $37.2 \%$ (95\% CI 26.5 , $48.9)$ in the standard care group (41.6\% RRR, $p=0.02)$. For women, these figures were $16.7 \%(95 \%$ CI $12.2,22.2)$ and $24.1 \%$ (95\% CI $17.3,32.0)$, respectively (30.6\% RRR, $p=0.08)$.

At the end of the first year of follow-up, $24.7 \%$ of individuals in the intensive group and $18.8 \%$ of those in the standard care group reduced their weight by at least $3 \%$. These percentages were $20.4 \% / 20.3 \%$ at the end of the second year, $20.5 \% / 17.6 \%$ at the end of the third and $19.6 \% / 14.7 \%$ at the end of the fourth year.

According to these results, 9.5 participants who choose to take part in the lifestyle intervention programme needed to be treated for 4 years to prevent one case of type 2 diabetes (NNT 9.5).

Results from the proportional hazards regression model indicated that hyperglycaemia was the most relevant predictive factor for developing diabetes (Table 3). The risk of developing diabetes at follow-up increased either with increasing baseline $\mathrm{HbA}_{1 \mathrm{c}}$ (HR 2.16 [95\% CI 1.25, 3.74]) or, especially, when prediabetes was present in any form: IFG (HR 3.56 [95\% CI 1.67, 7.56]), IGT (HR 5.37 [95\% CI $3.25,8.88]$ ) or both categories (HR 9.39 [95\% CI 4.89, 18.03]). The BMI (HR 1.08 per $\mathrm{kg} / \mathrm{m}^{2}$ [95\% CI 1.04, 1.12]) was also an independent predictive factor. Regarding preventive lifestyle intervention, the intensive approach had a protective effect on diabetes incidence (HR 0.54 [95\% CI $0.37,0.79])$ compared with the standard care intervention. Multivariate analysis evidenced no predictive value for age, sex and other classic risk factors evaluated, except for history of blood glucose disorders (HR 2.01 [95\% CI 1.37, 2.96]). When a new model was set up omitting biochemical 
Table 1 Baseline characteristics in the standard care $(n=219)$ and intensive intervention $(n=333)$ groups
Data are shown as mean \pm SD for continuous variables or $n(\%)$ for categorical variables

\begin{tabular}{|c|c|c|c|}
\hline Variable & $\begin{array}{l}\text { Standard care intervention } \\
\text { group }(n=219)\end{array}$ & $\begin{array}{l}\text { Intensive intervention } \\
\text { group }(n=333)\end{array}$ & $p$ value \\
\hline Age (years) & $62.0 \pm 7.9$ & $62.2 \pm 8.0$ & 0.79 \\
\hline Sex (women) & $141(64.4)$ & $227(68.2)$ & 0.36 \\
\hline FINDRISC score & $16.2 \pm 3.3$ & $15.8 \pm 3.6$ & 0.26 \\
\hline BMI $\left(\mathrm{kg} / \mathrm{m}^{2}\right)$ & $31.3 \pm 4.7$ & $31.2 \pm 4.7$ & 0.77 \\
\hline Waist circumference $(\mathrm{cm})$ & $101.6 \pm 11.1$ & $100.0 \pm 10.3$ & 0.08 \\
\hline Systolic BP (mmHg) & $135.2 \pm 14.3$ & $133.2 \pm 14.3$ & 0.11 \\
\hline Diastolic BP (mmHg) & $80.9 \pm 9.6$ & $79.7 \pm 8.9$ & 0.14 \\
\hline FPG (mmol/l) & $5.3 \pm 0.7$ & $5.2 \pm 0.7$ & 0.21 \\
\hline $2 \mathrm{hPG}(\mathrm{mmol} / \mathrm{l})$ & $7.1 \pm 2$ & $6.9 \pm 2$ & 0.27 \\
\hline Total cholesterol (mmol/l) & $5.4 \pm 0.9$ & $5.5 \pm 0.9$ & 0.80 \\
\hline HDL-cholesterol (mmol/1) & $1.5 \pm 0.4$ & $1.5 \pm 0.4$ & 0.55 \\
\hline LDL-cholesterol (mmol/1) & $3.3 \pm 0.8$ & $3.3 \pm 0.8$ & 0.35 \\
\hline Triacylglycerol (mmol/l) & $1.5 \pm 0.7$ & $1.4 \pm 0.8$ & 0.64 \\
\hline Prediabetes, $n(\%)$ & $103(47)$ & $148(44.4)$ & 0.55 \\
\hline $\mathrm{HbA}_{1 \mathrm{c}}(\%)(\mathrm{NGSP})$ & $5.31 \pm 0.35$ & $5.34 \pm 0.38$ & 0.26 \\
\hline $\mathrm{HbA}_{1 \mathrm{c}}(\mathrm{mmol} / \mathrm{mol})(\mathrm{IFCC})$ & $34.5 \pm 3.8$ & $34.9 \pm 4.1$ & 0.26 \\
\hline
\end{tabular}

measurements of glucose but keeping the FINDRISC items separate (Table 3), the intensive intervention retained its protective effect (HR 0.65 [95\% CI 0.45, 0.92]) and the history of blood glucose disorders gained in predictive value (HR 2.52 [95\% CI 1.76, 3.61]). In addition, the use of medication for high blood pressure (HR 1.52 [95\% CI $1.04,2.21])$ appeared as an independent outstanding predictor.

\section{Discussion}

Compelling evidence has been accumulated to support the efficacy of intensive lifestyle intervention to prevent or delay the incidence of type 2 diabetes [1]. It has also been shown that people identified as being at high risk of diabetes determined by the FINDRISC will significantly benefit from lifestyle intervention [11]. However, efficacy is not
Table 2 Baseline risk pattern characteristics of participants who progressed to diabetes $(n=124)$ or did not $(n=428)$ during the study
Data are shown as mean \pm SD for continuous variables or $n(\%)$ for categorical variables

\begin{tabular}{|c|c|c|c|}
\hline Variable & $\begin{array}{l}\text { Progressed to diabetes } \\
(n=124)\end{array}$ & $\begin{array}{l}\text { Did not progress to } \\
\text { diabetes }(n=428)\end{array}$ & $p$ value \\
\hline Age (years) & $62.6 \pm 7.7$ & $62 \pm 8$ & 0.49 \\
\hline Sex (women) & $72(58.1)$ & $296(69.2)$ & 0.02 \\
\hline FINDRISC score & $16.7 \pm 4.1$ & $15.8 \pm 3.2$ & 0.006 \\
\hline BMI $\left(\mathrm{kg} / \mathrm{m}^{2}\right)$ & $32.1 \pm 5.1$ & $30.9 \pm 4.5$ & 0.01 \\
\hline Waist circumference $(\mathrm{cm})$ & $103 \pm 11.6$ & $99.9 \pm 10.3$ & 0.005 \\
\hline Systolic BP (mmHg) & $136 \pm 16.2$ & $133.4 \pm 13.7$ & 0.09 \\
\hline Diastolic BP (mmHg) & $82.2 \pm 9.1$ & $79.5 \pm 9.1$ & 0.004 \\
\hline FPG (mmol/l) & $5.6 \pm 0.7$ & $5.1 \pm 0.7$ & $<0.001$ \\
\hline $2 \mathrm{hPG}(\mathrm{mmol} / \mathrm{l})$ & $8.5 \pm 1.7$ & $6.6 \pm 1.8$ & $<0.001$ \\
\hline Total cholesterol (mmol/l) & $5.4 \pm 0.9$ & $5.5 \pm 0.9$ & 0.69 \\
\hline HDL-cholesterol (mmol/l) & $1.4 \pm 0.3$ & $1.5 \pm 0.4$ & 0.01 \\
\hline LDL-cholesterol (mmol/l) & $3.3 \pm 0.9$ & $3.3 \pm 0.8$ & 0.93 \\
\hline Triacylglycerol (mmol/l) & $1.6 \pm 1.0$ & $1.4 \pm 0.7$ & 0.003 \\
\hline Prediabetes, $n(\%)$ & $102(82.3)$ & $149(34.8)$ & $<0.001$ \\
\hline $\mathrm{HbA}_{1 \mathrm{c}}(\%)(\mathrm{NGSP})$ & $5.51 \pm 0.37$ & $5.28 \pm 0.35$ & $<0.001$ \\
\hline $\mathrm{HbA}_{1 \mathrm{c}}(\mathrm{mmol} / \mathrm{mol})(\mathrm{IFCC})$ & $36.7 \pm 4.1$ & $34.2 \pm 3.8$ & $<0.001$ \\
\hline Intensive intervention, $\mathrm{n}(\%)$ & $61(49.2)$ & $272(63.6)$ & 0.004 \\
\hline
\end{tabular}


Fig. 2 Proportion of subjects without diabetes during the study and the $95 \%$ CI for the cumulative probability of remaining diabetes-free (lines) in the standard-care intervention group $(n=219$; white diamonds) and the intensive intervention group $(n=333$; black diamonds). $p=0.005$ (logrank)

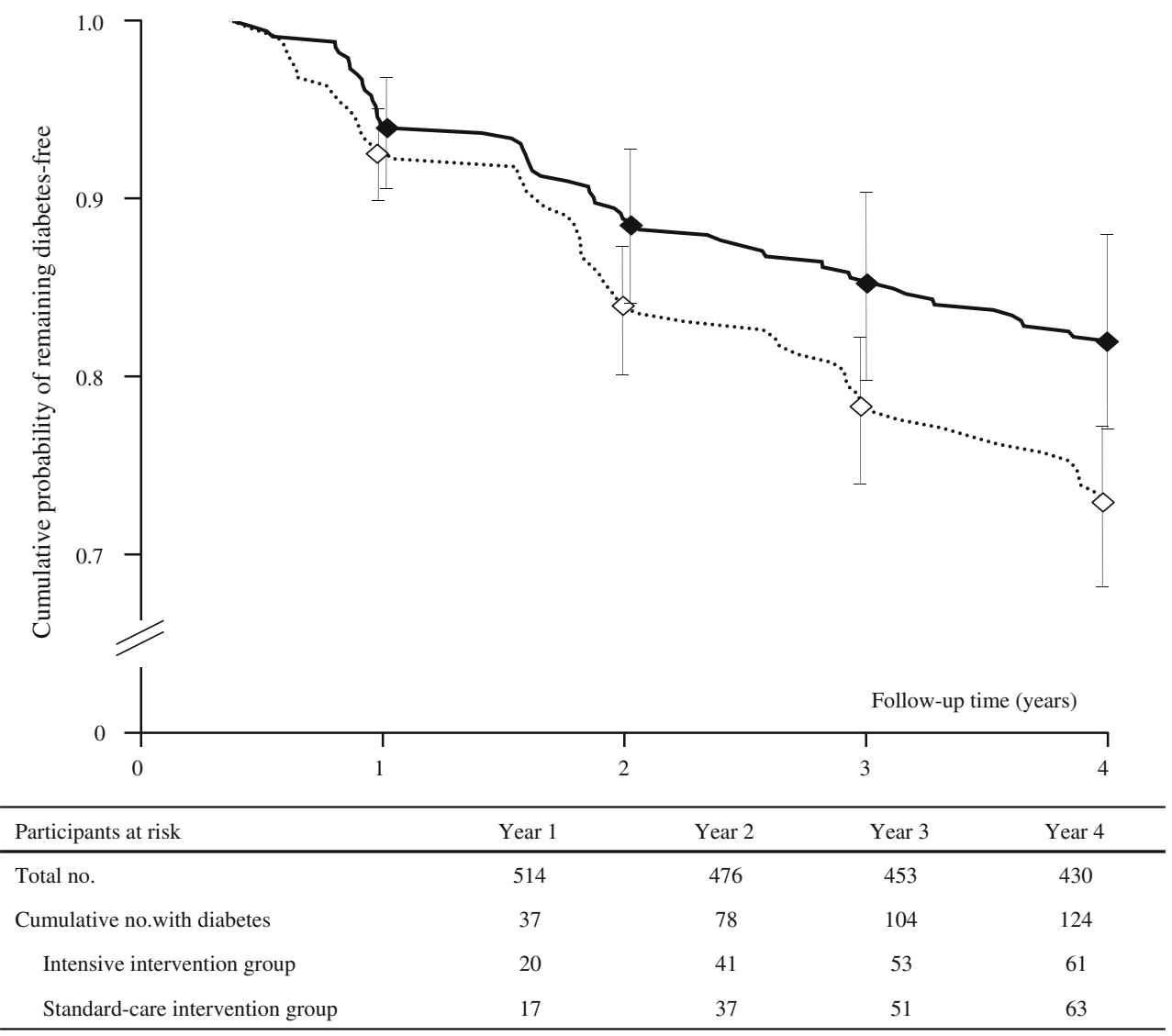

equivalent to effectiveness, in view of transferring the findings of diabetes prevention research into real life, particularly in primary healthcare. In fact, assessing effectiveness in diabetes prevention appears to represent a forced landing into reality [12] and implementation of clinical trial interventions into community settings has been difficult everywhere [11-15].

No wonder only a limited number of countries have projected national plans based on screening the population at risk and developing a continuous preventive intervention [2]. Obviously, this intervention has to be proven to be effective and even cost-effective [16]. The DE-PLAN project followed this course of action entirely within the scope of public health in Catalonia (nearly 7 million inhabitants).

If efficacy refers to the adequacy of the outcome (reducing the incidence of diabetes) to target (prevent or delay its onset), one could say categorically that lifestyle modification is an effective measure, based on published clinical trials. In contrast, if effectiveness means the real possibility of inserting proven measures in daily practice, one could say that no initiative has been shown truly effective in preventing type 2 diabetes. The term effectiveness seems appropriate to refer to the results of this study, whereas the first objective was to show that intensive lifestyle intervention was feasible in primary care, at least over 4 years. We have scheduled subsequent annual monitoring of the participants who did not develop diabetes. As far as we know, these data represent the first assessment of long-term feasibility and effectiveness of a public healthcare strategy to prevent type 2 diabetes within a European primary care setting.

Contrary to population-based studies, but similar to others conducted in communities or workplaces [14], this project essentially focused on a large sample of undiagnosed highrisk individuals, where the likelihood of developing glucose abnormalities and diabetes clearly increases. The feasibility and effectiveness of lifestyle interventions need to be validated within the population in which they are intended to be used. The Catalan DE-PLAN project evidenced that the overall incidence of diabetes was reduced by $36 \%$ at 4 -year followup in individuals following the intensive intervention compared with the standard care regime. This is a significant result with important implications for primary healthcare-based diabetes prevention. Although there are costs associated with such a prevention programme, 9.5 individuals needed-to-treat to reduce one case of diabetes is a promising index regarding cost-effectiveness of the intensive intervention when compared with the standard care one [16]. The design of the associated cost-effectiveness analysis has been already published [17].

The reduction in diabetes incidence achieved with intensive lifestyle modification in this study of $36 \%$ was more modest than that observed in trials, which have shown a $58 \%$ reduction in diabetes incidence $[18,19]$. The decline in 
Table 3 Independent determinants of developing type 2 diabetes, from the proportional hazards model analysis

\begin{tabular}{lll}
\hline Variable & $\begin{array}{l}\text { Including all } \\
\text { biochemical glucose } \\
\text { measurements }\end{array}$ & $\begin{array}{l}\text { Omitting all } \\
\text { biochemical glucose } \\
\text { measurements }\end{array}$ \\
\hline BMI $\left(\mathrm{kg} / \mathrm{m}^{2}\right)$ & $1.08(1.04,1.12)$ & - \\
$\begin{array}{l}\text { History of blood } \\
\text { glucose disorders }\end{array}$ & $2.01(1.37,2.96)$ & $2.52(1.76,3.61)$ \\
IFG & $3.56(1.67,7.56)$ & - \\
IGT & $5.37(3.25,8.88)$ & - \\
$\begin{array}{l}\text { Both conditions } \\
\text { IFG+IGT) }\end{array}$ & $9.39(4.89,18.03)$ & - \\
$\begin{array}{l}\text { HbA } 1 \mathrm{c} \text { (per 1\%) } \\
\text { Intensive intervention }\end{array}$ & $2.16(1.25,3.74)$ & - \\
$\begin{array}{l}\text { Use of antihypertensive } \\
\text { drugs }\end{array}$ & - & $0.54(0.37,0.79)$ \\
\hline
\end{tabular}

Data are shown as HR and the corresponding 95\% CI

All variables with independent predictive value are presented. The analyses were adjusted for lifestyle intervention group, sex, age and baseline blood test results. All biochemical glucose measurements or the corresponding diagnostic category of prediabetes were included in the first model and then omitted in the second, keeping the FINDRISC items separately

the incidence of diabetes was progressive, starting immediately after implementing the intervention, reaching a statistically significant difference by the third year and then continuing until the study close-out. The difference in effect with respect to that obtained in academic clinical trials can be explained easily by differences in methods and intensity of interventions applied. The core intervention programme implemented in Catalonia was similar to that applied in the Finnish Diabetes Prevention Study [5, 18] following the practice and evidence-based guideline for diabetes prevention as part of the European IMAGE project $[3,4]$. The transfer of such interventions was conducted in community settings, administered through an intensive education programme and delivered exclusively by trained primary healthcare professionals. However, the primary care intervention was projected to search for changes not only in participant lifestyles but also in the clinical practice of participating centres.

When comparing this study with clinical trials, the most important limitation is the process of allocation to intervention and we cannot rule out some selection bias. In fact, the DE-PLAN was never defined as a clinical trial, but as a public health study; and group assignment was not handled by a randomisation list, but by successive entry, and participants' preference as to type of intensive intervention was ultimately respected. It is likely that the effect of the intervention may have been overestimated if exclusively referred to this type of bias. But there is another, opposite, bias at work. The data were analysed according to the intention-totreat principle, even though some intensive intervention group participants did not follow the recommendations. In contrast, all participants in the standard care group received general health advice at baseline and at follow-up visits and may have benefited from these recommendations. If this type of bias is also considered, the effect of the intervention may have been underestimated.

Another limitation is the relatively high discontinuation rate. Even accepting the technical difficulties in measuring the participants' motivation to make lifestyle changes and also assuming that there were no large differences between the participants assigned to either standard or intensive lifestyle interventions, the discontinuation throughout the study was somewhat higher in the standard care group. Undoubtedly, the main reason is that the protocol was conducted under real working conditions. To increase effectiveness, methodological issues surrounding the implementation and comparison of programmes carried out under standard care conditions have to be explored. That is why we are currently conducting a thorough analysis on changes in participants' lifestyle habits by type of intervention applied. As published, a sustained effect can be possible based on the people participating in the intervention itself [20]; nevertheless, we suggest that the continued reinforcement by the primary care teams also influenced our study results.

The general profile of the participants was similar to that of the primary care-attended population. In fact, women are the ones who most often use these services in Spain [21, 22] and such predominance is similar to both Finnish and American trials published $[18,19]$. It seems that individuals identified as at high risk at screening all benefit similarly from lifestyle intervention, regardless of age and sex. In previous controlled trials, older people seemed to benefit somewhat more than younger ones, but men and women both had similar outcomes. The Finnish trial revealed that there was no difference in the benefit from lifestyle intervention among different socioeconomic groups [23]. Consequently, in specifying the target participant profile for diabetes prevention in primary care, it is not necessary to pay too much attention to population subgroups; however, it is important to plan properly for consistent preventive measures, if possible at the national level [24].

The study also provided evidence of the direct (prediabetes diagnosed at the screening) and indirect $\left(\mathrm{HbA}_{1 \mathrm{c}}\right)$ independent predictive role of hyperglycaemia on diabetes incidence. In addition, BMI and previous history of high glucose disorders were independent predictors. Nevertheless, the intensive lifestyle intervention approach had a protective effect on incidence compared with the standard care approach in all multivariate models performed.

Several risk scores predict diabetes risk based on noninvasive routine measures or on data from questionnaires [25]. Therefore, substantially reducing diabetes incidence is 
feasible in real-life primary healthcare settings using intensive lifestyle intervention among high-risk individuals identified first with a simple method such as the FINDRISC tool. Our recommendation is that all people attending primary healthcare facilities should be screened for the risk of diabetes. Intervention programmes that have now been developed and scientifically tested, not only by academic clinical trials but by primary care implementations, must be recognised as the standard evidence-based healthcare. It would consequently be helpful to include these conclusions in the European guideline for type 2 diabetes prevention.

Acknowledgements In 2010, the project received the first prize for innovation and organisation in primary healthcare processes from the Department of Health and Catalan societies of medicine (CAMFiC) and nursing (AIFICC). A part of this study was accepted for presentation at the 2010 and 2011 European Association for the Study of Diabetes meetings. À. Sabaté (Centre Diabetis Salou, Tarragona, Spain) and Pharmalexic-Barcelona, provided English writing assistance and received professional fees for their work.

Funding This project was funded by the Commission of the European Communities, Directorate C - Public Health, grant agreement no. 2004310, by the Institute of Health Carlos III, Spanish Ministry of Health, grant agreements FIS (PI05-033 and PS09-001112), and the Department of Health, Generalitat de Catalunya.

Duality of interest statement The authors declare that there is no duality of interest associated with this manuscript.

Contribution statement All authors substantially contributed to designing the study protocol or to data analysis and interpretation, as well as to drafting or revising the article. Specifically, BC wrote the national research proposal and the core of this manuscript; JLP and FB performed the statistical analysis; JS, OSM, JJC, XC, JL and JT added significant contributions; $\mathrm{CC}, \mathrm{BB}$ and JB organised the study within the structure of our public health system and CS coordinated the participation of nursing and the development of lifestyle intervention. All authors read and approved the final version to be published.

\section{Appendix}

Members of the DE-PLAN-CAT Coordinating Committee: M. Álvarez, F. Barrio, B. Bolíbar, J.-L. Bueno, M. Bulló, J.-J. Cabré, A. Castaño, C. Castell, J.-À. Caula, X. Cos, B. Costa, M. Cot, J.-A. Fernández, L. González, J. Jurado, T.-M. Llauradó, X. Mundet, T. Mur, I. Ojeda, M. Ortigas, M. Pastoret, J.-L. Piñol, F. Pujol, M. Roura, R. Sagarra, J. Salas, S. Sarret, C. Solé and M. Torres.

\section{References}

1. Gillies CL, Abrams KR, Lambert PC et al (2007) Pharmacological and lifestyle interventions to prevent or delay type 2 diabetes in people with impaired glucose tolerance: systematic review and meta-analysis. BMJ 334:229-237
2. Alberti KG, Zimmet P, Shaw J (2007) International Diabetes Federation: a consensus on type 2 diabetes prevention. Diabet Med 24:451-463

3. Paulweber B, Valensi P, Lindström et al (2010) A European evidence-based guideline for the prevention of type 2 diabetes. Horm Metab Res 42(Suppl 1):S3-S36

4. Lindström J, Neumann A, Sheppard KE et al (2010) Take action to prevent diabetes - the IMAGE toolkit for the prevention of type 2 diabetes in Europe. Horm Metab Res 42(Suppl 1):S37-S55

5. Schwarz PE, Lindström J, Kissimova-Scarbeck K et al (2008) The European perspective of type 2 diabetes prevention: diabetes in Europe - prevention using lifestyle, physical activity and nutritional intervention (DE-PLAN) project. Exp Clin Endocrinol Diabetes 116:167-172

6. Lindström J, Tuomilehto J (2003) The Diabetes Risk Score: a practical tool to predict type 2 diabetes risk. Diabetes Care $26: 725-731$

7. WHO (1999) Definition, diagnosis, and classification of diabetes mellitus and its complications: report of a WHO consultation. Part 1. Diagnosis and classification of diabetes mellitus. World Health Organization, Geneva, pp 1-59

8. The International Expert Committee (2009) International Expert Committee Report on the role of the A1c assay in the diagnosis of diabetes. Diabetes Care 32:1327-1334

9. Schröder H, Fitó M, Estruch R et al (2011) A short screener is valid for assessing Mediterranean diet adherence among older Spanish men and women. J Nutr 141:1140-1145

10. Costa B, Piñol J, Martín F, Donado A, Castell C (2002) Significant incidence of type 2 diabetes on high-risk Spanish population. The IGT Study (2). Med Clin (Barc) 118:287-293

11. Saaristo T, Moilanen L, Korpi-Hyövälti E et al (2010) Lifestyle intervention for prevention of type 2 diabetes in primary health care: one-year follow-up of the Finnish National Diabetes Prevention Program (FIN-D2D). Diabetes Care 33:2146-2151

12. Costa B (2008) The effectiveness in type 2 diabetes prevention: a forced landing to reality. Med Clin (Barc) 130:295-297

13. Makrilakis K, Liatis S, Grammatikou S, Perrea D, Katsilambros N (2010) Education and psychological aspects implementation and effectiveness of the first community lifestyle intervention programme to prevent type 2 diabetes in Greece. The DE-PLAN study. Diabet Med 27:459-465

14. Sakane N, Sato J, Tsushita K et al (2011) Prevention of type 2 diabetes in a primary healthcare setting: three-year results of lifestyle intervention in Japanese subjects with impaired glucose tolerance. BMC Public Health 11:40-47

15. Katula JA, Vitolins MZ, Rosenberger EL et al (2011) 1-year results of a community-based translation of the diabetes prevention program: Healthy-Living Partnerships to Prevent Diabetes (HELP PD) project. Diabetes Care 34:1451-1457

16. Herman WH (2011) The economics of diabetes prevention. Med Clin N Am 95:373-384

17. Costa B, Cabré JJ, Sagarra R et al (2011) Rationale and design of the PREDICE project: cost-effectiveness of type 2 diabetes prevention among high-risk Spanish individuals following lifestyle intervention in real-life primary care setting. BMC Public Health $11: 623-629$

18. Tuomilehto J, Lindström J, Eriksson JG et al (2001) Prevention of type 2 diabetes mellitus by changes in lifestyle among subjects with impaired glucose tolerance. N Engl J Med 344:1343-1392

19. Diabetes Prevention Program Research Group (2002) Reduction in the incidence of type 2 diabetes with lifestyle intervention or metformin. N Engl J Med 346:393-403

20. Lindström J, Ilanne-Parikka P, Peltonen M et al (2006) Sustained reduction in the incidence of type 2 diabetes by lifestyle intervention: follow-up of the Finnish Diabetes Prevention Study. Lancet $368: 1673-1679$ 
21. Soler-González J, Serna-Arnáiz C, Rué-Monné M, Bosch-Gaya A, Ruiz-Magaz MC, Gervilla-Caño J (2008) Use of primary care resources by immigrants and the autochthonous persons who contact the care services in the city of Lleida, Spain. Aten Primaria 40:225-233

22. Calderón-Larrañaga A, Gimeno-Feliu LA, Macipe-Costa R, Poblador-Plou B, Bordonaba-Bosque D, Prados-Torres A (2011) Primary care utilisation patterns among an urban immigrant population in the Spanish National Health System. BMC Public Health 11:432-439
23. Diabetes Prevention Program Research Group (2009) 10-year follow-up of diabetes incidence and weight loss in the Diabetes Prevention Program Outcomes Study. Lancet 374:1677-1686

24. Rautio N, Jokelainen J, Oksa H et al (2011) Socioeconomic position and effectiveness of lifestyle intervention in prevention of type 2 diabetes: one-year follow-up of the FIN-D2D project. Scand J Public Health 39:561-570

25. Buijsse B, Simmons RK, Griffin SJ, Schulze MB (2011) Risk assessment tools for identifying individuals at risk of developing type 2 Diabetes. Epidemiol Rev 33:46-62 